\title{
An influenza A virus vaccine based on an M2e-modified alphavirus
}

\author{
Haritha Ramya Krishnavajhala $^{1} \cdot$ Jacqueline Williams $^{1} \cdot$ Hans Heidner $^{1}$
}

Received: 31 July 2017 / Accepted: 11 September 2017 / Published online: 27 October 2017

(c) Springer-Verlag GmbH Austria 2017

\begin{abstract}
The 23-residue external domain of the influenza A virus M2 protein (M2e) has significant potential as a vaccine antigen. Here, we describe the construction and characterization of an M2e-modified Sindbis virus designated E2S1-M2e. E2S1-M2e virions contain M2e as an N-terminal extension of the E2 glycoprotein and therefore express 240 copies of the M2e peptide on their surface. The E2S1-M2e virus expressed $\mathrm{M} 2 \mathrm{e}$ in an accessible and immunogenic form and induced M2e-specific antibodies when administered to mice. Mice that received an intranasal vaccination with E2S1-M2e were protected against a lethal challenge with a virulent, mouse-adapted strain of influenza A virus.
\end{abstract}

Influenza A viruses are separated into subtypes based on the antigenic characteristics of the viral glycoproteins called hemagglutinin (HA) and neuraminidase (NA). Currently, 18 distinct HA types (HA1-HA18) and 11 distinct NA types (NA1-NA11) have been identified [29]. HA is the primary viral antigen present in current influenza vaccines, and immunological protection against infection by influenza A virus correlates with the presence of antibodies that inhibit HA activity and neutralize virus infectivity [13]. Immunity to influenza A virus tends to be narrow and strain-specific [10], and current vaccines include selected strains of only two influenza A virus subtypes (H1N1 and H3N2). The antigenic characteristics of HA change rapidly over time through the process of antigenic drift,

Handling Editor: Hans Dieter Klenk.

Hans Heidner

hans.heidner@utsa.edu

1 Department of Biology, The University of Texas at San Antonio, One UTSA Circle, San Antonio, TX 78006, USA and as a consequence, immunity induced by current vaccines often fails to offer protection against antigenically altered homotypic viruses that appear in subsequent flu seasons. In addition, these vaccines do not provide protection against heterotypic viruses or against novel pandemic virus strains [10]. Therefore, influenza virus vaccines must be reformulated each year in an effort to include the most relevant circulating strains of the virus, and individuals are encouraged to be revaccinated every year. The reformulation process is costly, delays vaccine production, and does not guarantee that the proper epidemic strains of the virus will be represented in the vaccine [16]. These problems have inspired the search for alternative vaccine antigens, and the influenza virus M2 protein has emerged as a promising candidate.

The M2 protein is an integral membrane protein that is expressed on the surface of mature virions [32] and on the surface of virus-infected cells [11]. M2 forms homotetrameric structures [6,27], which function as $\mathrm{pH}$-activated ion channels that transport hydrogen ions across the membrane $[19,25,26]$. This process plays an important role in cell entry [5] and in virion maturation [23, 28]. In contrast to the highly variable HA protein, M2 is relatively well conserved among strains of influenza A virus that have circulated in humans since 1918, and unlike HA, it does not experience a significant rate of antigenic drift [7,20]. Natural infection with influenza A virus does not induce a strong antibody response to M2 [2]; however, M2-specific antibodies can be produced at high levels when M2 is administered as a component of a vaccine. Most M2-based experimental vaccines have focused on the 23-residue external domain of the protein (M2e) [9]. Antibodies directed to M2/M2e do not neutralize virus infectivity, but some do limit virus spread and confer protection against a broad range of virus strains 
via Fc-dependent effector mechanisms (reviewed in reference [9]).

Many strategies have been used to enhance the immunogenicity of M2e-based vaccines. Collectively, these studies have demonstrated that the immunogenicity and efficacy of M2e-based vaccines can be enhanced considerably by increasing the M2e epitope density of the vaccine (reviewed in reference [9]). Here, we describe the construction and characterization of an influenza vaccine based on a replication-competent M2e-modified Sindbis virus (family Togaviridae, genus Alphavirus). The structure of the alphavirus particle is well suited for the presentation of peptide sequences at high density. The surface of the alphavirus particle contains 80 spikes [17, 30], each consisting of three identical heterodimers composed of glycoproteins E1 and E2 [21]. The M2e-modified Sindbis virus constructed for this study contains M2e as an N-terminal extension of the E2 glycoprotein, and therefore, each virion is predicted to express 240 copies of the M2e peptide on its surface.

The parental strain of Sindbis virus used in this study is designated E2S1 [3]. E2S1 contains two phenotypically silent mutations compared to the consensus sequence of the AR339 strain [8]. The genetic sequence encoding M2e was based on the sequence of the A/Puerto Rico/8/34 (H1N1) virus (PR8). The sequence of the encoded peptide is SLLTEVETPIRNEWGCRCNGSSD [15]. An overlapping polymerase chain reaction (PCR) cloning strategy was used to amplify the DNA sequences encoding the M2e peptide and to insert them into an infectious cDNA clone designated pTRSB-E2S1-ND/SK [8]. This procedure placed the M2e sequences in-frame between the sequences encoding $\mathrm{E} 3$ and E2 and immediately downstream of a furin cleavage site. The recombinant cDNA clone was designated pE2S1-M2e, and virus derived from the clone was designated E2S1-M2e. As designed, E2S1-M2e should express a modified PE2 glycoprotein that contains the M2e peptide sequence positioned between E3 and E2, with M2e fused to E2 through a flexible 17 residue $\left(\mathrm{RS}\left[\mathrm{G}_{4} \mathrm{~S}\right]_{3}\right.$ ) spacer (Fig. 1A). Furin-mediated cleavage of the modified PE2 protein should generate E3 and a protein containing the M2e peptide as an N-terminal extension of E2. The decision to insert the M2e sequence between E3 and E2 was based on prior studies, which demonstrated that foreign sequences inserted at this site were tolerated by the virus $[8,12]$ and that protein sequences expressed in this manner are presented on the virion surface in an accessible form $[8,14,18]$.

Infectious virus was derived from $\mathrm{pE} 2 \mathrm{~S} 1$ and $\mathrm{pE} 2 \mathrm{~S} 1-$ $\mathrm{M} 2 \mathrm{e}$, and the specific infectivity of virions was determined as described previously [3, 4]. The growth rates of E2S1 and E2S1-M2e were compared in BHK-21 cells using one-step growth curves [4]. E2S1-M2e was viable and replicated to near-normal titers and with normal kinetics (Fig. 1B). The reduced titers of E2S1-M2e compared to E2S1 are partially due to a slight reduction in particle infectivity, as the ratio of specific infectivity values for E2S1 and E2S1-M2e was calculated to be 1.0:0.6. E2S1-M2e retained a small-plaque phenotype compared to E2S1 after passage in cultured cells, suggesting that the virus is genetically stable and is not under a strong selective pressure to delete the M2e insert.

E2S1 and E2S1-M2e virions were radiolabeled during growth in BHK-21 cells, purified from cell supernatants and resolved by SDS-PAGE as described [1, 4]. This analysis demonstrated that the M2e-E2 fusion protein was incorporated into E2S1-M2e virions (Fig. 1C). The accessibility of $\mathrm{M} 2 \mathrm{e}$ on the E2S1-M2e virion surface was then confirmed by ELISA. E2S1 and E2S1-M2e virions were purified as described above, diluted in coating buffer ( $\mathrm{pH} 9.6)$, and bound to 96-well ELISA plates (Pro-Bind, Falcon) at 100 ng/well. Wells were washed repeatedly and blocked with $3 \%$ bovine serum albumin fraction $\mathrm{V}$ in PBS. Plates were washed and then probed with primary monoclonal antibodies specific for M2e (MAb 14C2, Santa Cruz Biotechnology, Santa Cruz, CA) [32], for Sindbis virus protein E2 (MAb 49) [24], and for bluetongue virus protein VP7 (MAb 290) [31]. MAbs were diluted 1:50 in blocking buffer, added to duplicate wells, and then processed in a twofold dilution series. Plates were incubated with primary MAbs for 1 hour, washed, and then probed with a secondary antibody (HRP-conjugated goat anti-mouse Ig H+L, Southern Biotechnology, Birmingham, AL) diluted 1:4000. Plates were incubated with the secondary antibody for 1 hour and then washed. OPD substrate was then added to each well and plates were incubated for 20 minutes. $\mathrm{OD}_{450}$ readings were then obtained, and ELISA titers were reported as the inverse of the highest $\mathrm{MAb}$ dilution that yielded an $\mathrm{OD}_{450}$ of $\geq 0.2$. MAb 14C2 failed to bind E2S1 virions but bound well to E2S1-M2e virions. MAb 49 bound equally well to both viruses, and the control antibody (MAB 290) failed to bind either virus (Fig. 1D).

The immunogenicity of E2S1 and E2S1-M2e was then evaluated in four groups (eight mice/group) of age-matched, 5- to 7-week-old, female BALB/c mice (Charles River Laboratories, Wilmington, MA). Prior to immunization, mice were anesthetized by inhalation of $3 \%$ isoflurane. Mice were then immunized by intranasal (IN) or subcutaneous (SC) administration of purified virions in saline at a dose of $1 \times 10^{3}$ or $1 \times 10^{6}$ PFU of virus. Mice were boosted similarly 21 days later. Two additional groups of mice (4 mice/group) were mock vaccinated with PBS by either the IN or SC route. Consistent with prior studies using Sindbis virus strain AR339 in immunocompetent adult mice [22], none of the virus-exposed mice displayed any visible signs of clinical infection. Blood samples were collected prior to vaccination and at day 42 by submandibular puncture using a 5-mm lancet ( $\leq 180 \mu \mathrm{l} \mathrm{blood/mouse).} \mathrm{Serum} \mathrm{content} \mathrm{of}$ blood was collected and stored at $-80{ }^{\circ} \mathrm{C}$. Bronchoalveolar 
A

E2S1
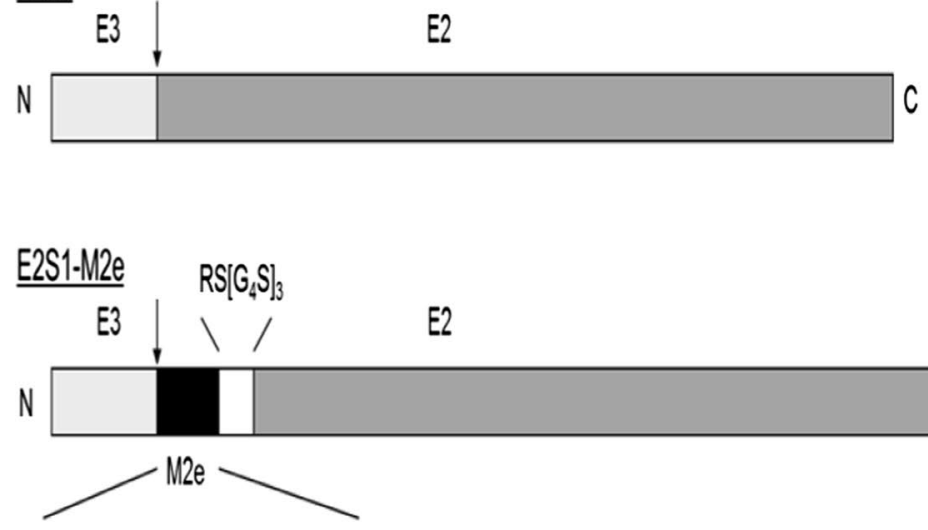

SLLTEVETPIRNEWGCRCNGSSD

C

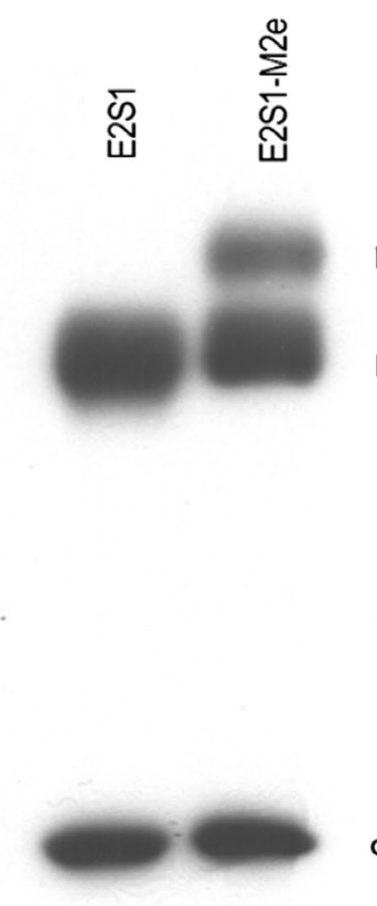

M2e-E2

E1/E2

capsid

Fig. 1 Characterization of the E2S1-M2e virus. A. Comparison of the PE2 proteins encoded by E2S1 and E2S1-M2e. The arrow indicates the furin cleavage site. B. Growth kinetics of E2S1 and E2S1M2e in BHK-21 cells. C. Protein composition of purified radiola-

lavage (BAL) fluids were collected from mice that were euthanized by $\mathrm{CO}_{2}$ asphyxiation followed by cervical dislocation. The trachea was exposed surgically and nicked with a scalpel. A $20 \mathrm{G}$ catheter was then inserted through the nick and eased towards the lungs. A single lavage was performed by injecting, and then withdrawing $1.0 \mathrm{ml}$ of PBS into the lungs using a tuberculin syringe. BAL fluids were clarified by microcentrifugation and frozen at $-80^{\circ} \mathrm{C}$.
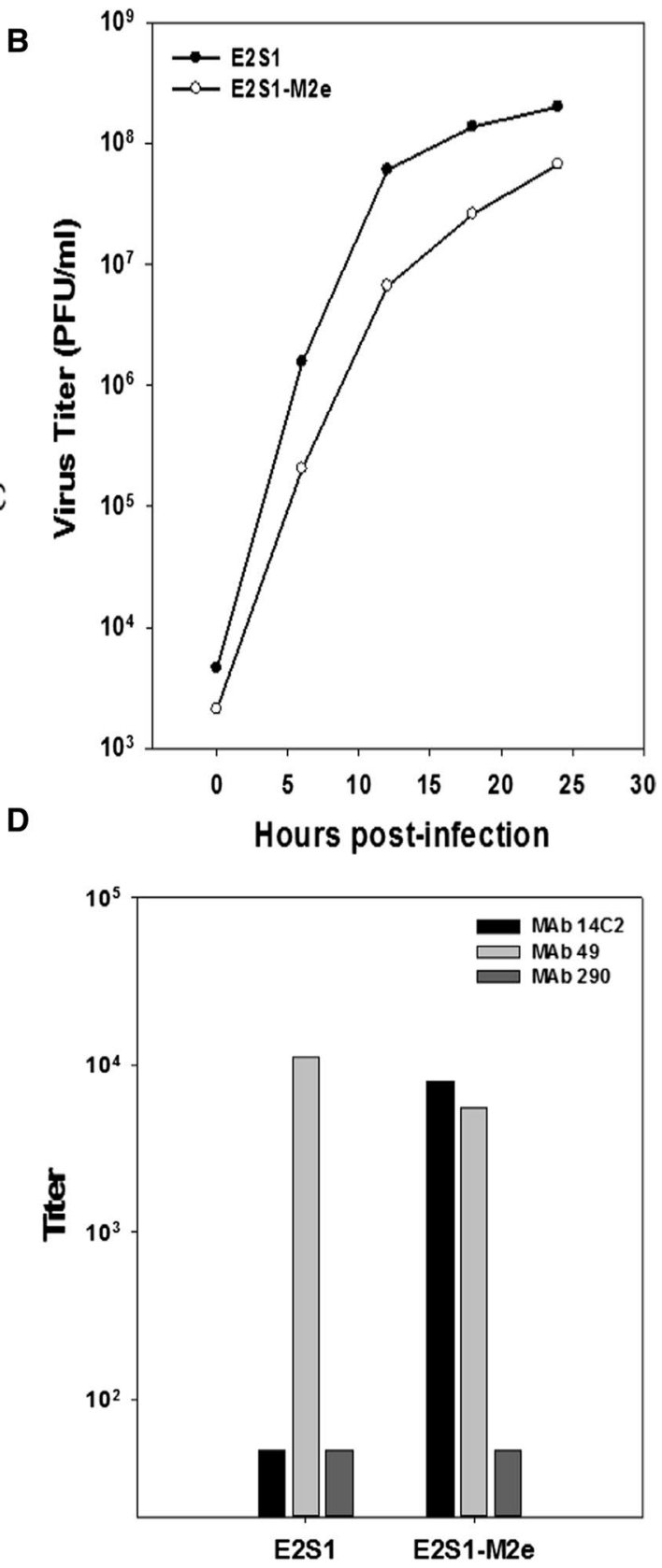

beled E2S1 and E2S1-M2e virions as determined by SDS-PAGE. D. Reactivity of E2S1 and E2S1-M2e virions with monoclonal antibodies specific for M2e (MAb 14C2), E2 (MAb 49), and bluetongue virus VP7 (MAb 290) using ELISA as described in the text

Serum and BAL samples were evaluated for antibodies to Sindbis virus antigens and to M2e by ELISA. E2S 1 virions were purified from infected cell supernatants, quantified by Bradford protein assay, and bound to ELISA plates at a concentration of $100 \mathrm{ng} /$ well. ELISA plates were also coated with a synthetic M2e peptide (GenScript, Piscataway, NJ) at a concentration of $50 \mathrm{ng} / \mathrm{well}$. Mouse serum or BAL fluids served as the sources of primary 
antibodies in these assays, and an HRP-conjugated goat anti-mouse $\mathrm{Ig} \mathrm{H}+\mathrm{L}$ was used as the secondary antibody. Primary antibody samples were processed in a twofold dilution series starting at dilutions of 1:50 (serum) or 1:4 (BAL fluid). ELISA titers were determined as described above. Mice exposed to E2S1 and E2S1-M2e produced similar antibody responses (total $\mathrm{Ig}$ ) to viral antigens, but only the mice that received E2S1-M2e produced serum and BAL antibodies (total Ig) specific for the M2e peptide (Fig. 2). The SC and IN routes of vaccination appeared to
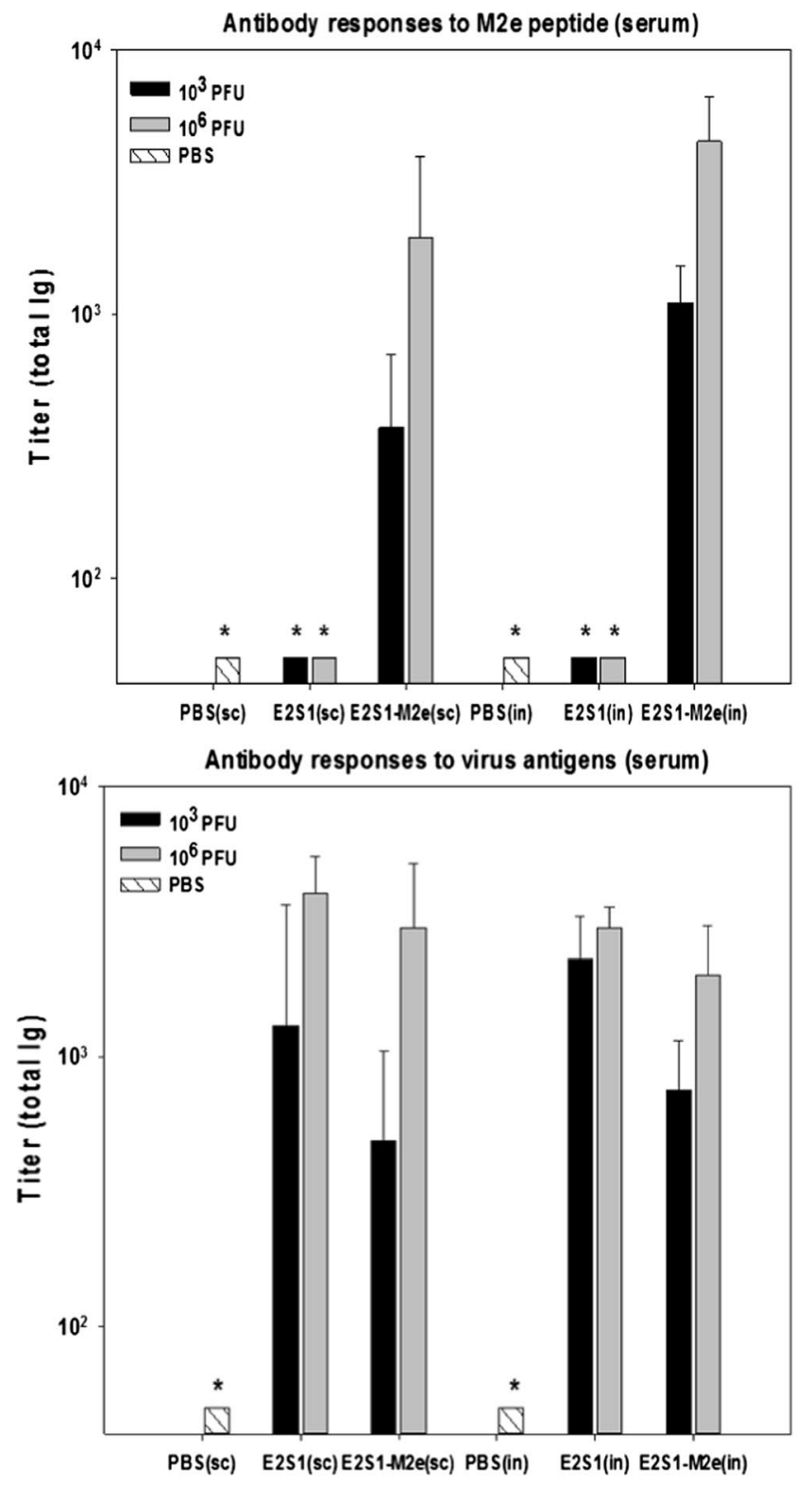

Fig. 2 Immunogenicity of E2S1 and E2S1-M2e in vaccinated mice. Groups of mice (eight mice/group) were vaccinated with low dose $\left(10^{3} \mathrm{PFU}\right)$ or high dose $\left(10^{6} \mathrm{PFU}\right)$ of E2S1 or E2S1-M2e administered by the intranasal (in) or subcutaneous (sc) route. Groups of control mice (four mice/group) were mock vaccinated with PBS. be equally effective for inducing virus- and M2e-specific antibodies in the serum and BAL (Fig. 2).

Based on the results of this immunogenicity study, a vaccination/challenge experiment was performed. Groups of mice (eight mice/group) were vaccinated as described above with $10^{6} \mathrm{PFU}$ of E2S1 or E2S1-M2e via the IN route and boosted similarly on day 21 . A control group of eight mice received PBS. All mice (except four PBS control mice), were then challenged on day 42 with a virulent, mouse-adapted strain of influenza A virus (PR8). The four control mice were
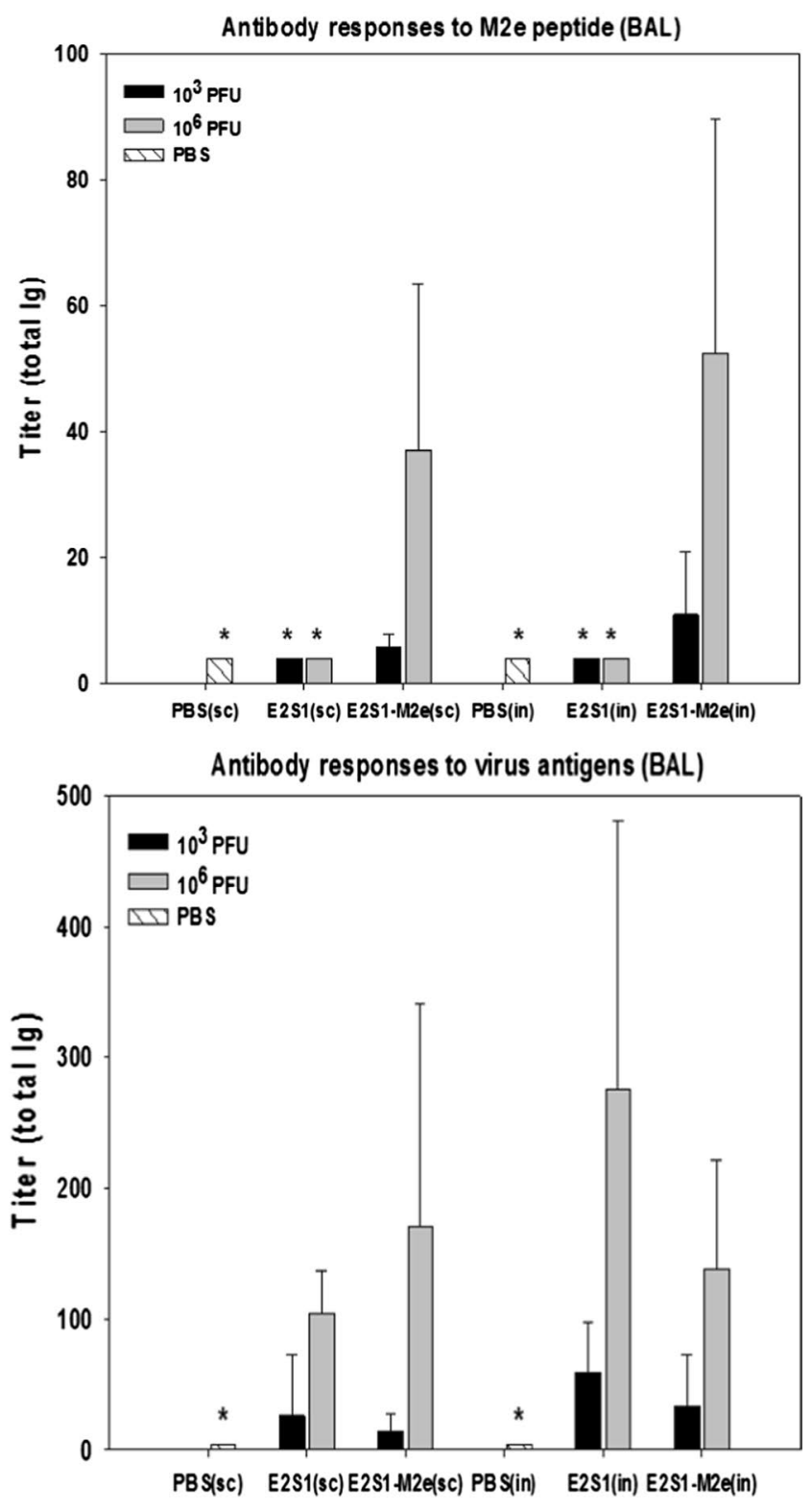

Animals were boosted similarly on day 21. Serum and BAL samples were collected on day 42. Antibody responses (serum and BAL) to viral antigens and to M2e were then assessed by ELISA. *, OD $450 \mathrm{~nm}$ $\leq 0.2$ at the lowest dilution tested (1:50 for serum and 1:4 for BAL) 


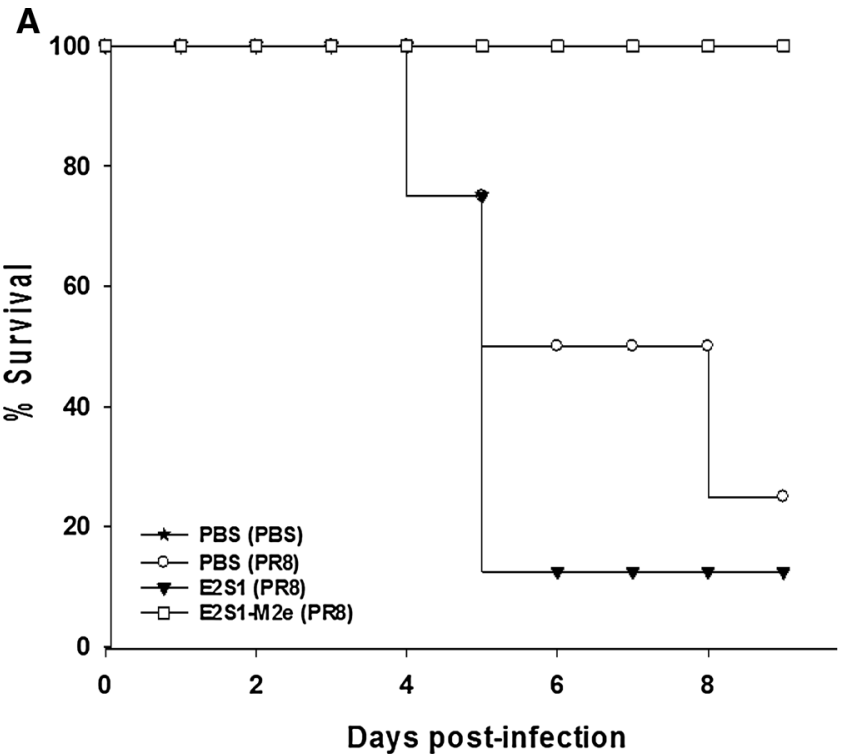

Fig. 3 Protective efficacy of E2S1-M2e in mice following a lethal challenge with influenza A virus. Groups of mice (eight mice/group) were vaccinated by the IN route with E2S1 $\left(10^{6} \mathrm{PFU}\right)$, E2S1-M2e $\left(10^{6} \mathrm{PFU}\right)$, or PBS, and then boosted similarly on day 21 . On day 42 , all mice vaccinated with E2S1 or E2S1-M2e, and four of the PBS

mock challenged with PBS. Prior to challenge, mice were anesthetized and weighed. Mice then received 500 PFU of PR8 virus in $50 \mu \mathrm{l}\left(10 \mathrm{LD}_{50}\right)$ administered by the IN route. Mice were weighed every 12 hours for 9 days and mice that lost $20 \%$ of their body weight were euthanized by $\mathrm{CO}_{2}$ asphyxiation followed by cervical dislocation. Four control mice (PBS used for mock vaccination and mock challenge) and all mice vaccinated with E2S1-M2e survived challenge (Fig. 3A) and displayed minimal loss of weight (Fig. 3B). In contrast, seven of eight mice vaccinated with E2S1 and three of four control mice (PBS used for mock vaccination followed by PR8 challenge) had to be euthanized (Fig. 3A). The single surviving mouse in each of these groups maintained weight after being challenged and displayed no clinical signs of illness. Based on the rapid weight loss and obvious clinical signs displayed by all other mice in their groups (hunching, ruffled fur, inactivity), we suspect that these two mice did not receive the intended dose of challenge virus. Several animals sneezed immediately following administration of virus into their nares, likely expelling most of the challenge virus. Data collected on these two animals are not included in Fig. 3B.

Strategies to enhance the epitope density of M2e in experimental vaccines have included the linking of tandem copies of M2e, linking M2e peptides onto synthetic scaffolds, and expressing the M2e peptide on the surface of phages, plant viruses or virus-like particles (reviewed in reference [9]). To our knowledge, E2S1-M2e represents

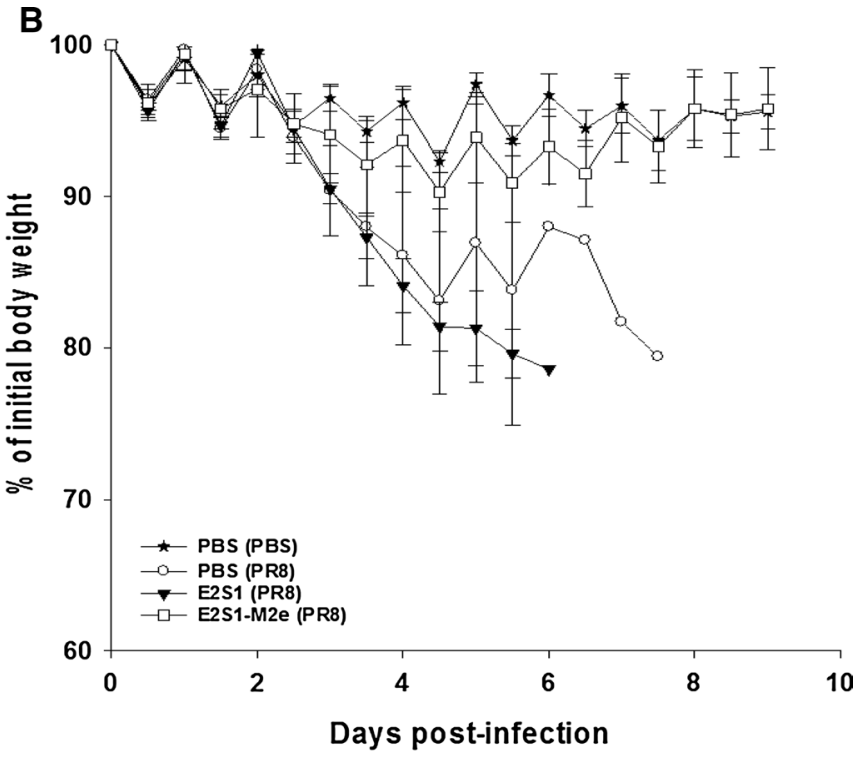

mock-vaccinated mice were challenged IN with influenza A virus (10 $\mathrm{LD}_{50}$ of PR8). Four of the mock-vaccinated mice (PBS) were mock challenged with PBS. A. Survival rates of mice following challenge. B. Body weights of challenged animals monitored every 12 hours for 9 days

the first M2e-based vaccine constructed as an M2e-modified virus capable of replicating within the vaccinated vertebrate host. Based on what is known about previously characterized M2e-based vaccines, it is reasonable to assume that the protective effects of E2S1-M2e are due in part to the induction of M2e-specific antibodies that restrict the replication of influenza A virus through $\mathrm{Fc}$ dependent immune mechanisms. However, M2e-specific $\mathrm{CD}^{+}$and $\mathrm{CD}^{+} \mathrm{T}$ cell responses may also contribute to protection against influenza A virus [9], and future studies will investigate the ability of E2S1-M2e to induce antiviral $\mathrm{T}$ cell responses and will investigate the extent to which immune responses to E2S1-M2e restrict the replication, spread, and pathogenesis of influenza A virus in the lung. In addition, Sindbis virus replicates well in avian species, including chickens, without causing clinical disease, and it will be of interest to evaluate the immunogenicity and protective efficacy of E2S1-M2e in this species.

Acknowledgements The PR8 virus was kindly provided by Dr. Mark Heise, University of North Carolina at Chapel Hill.

\section{Compliance with ethical standards}

Conflict of interest The authors have no conflict of interest to declare.

Research involving human participants and/or animals All applicable international, national, and/or institutional guidelines for the care and use of animals were followed. 
Informed consent This research project did not include any human participants.

Funding Funding for this project was provided by the University of Texas at San Antonio's Collaborative Research Seed Grant Program.

\section{References}

1. Boehme KW, Williams JC, Johnston RE, Heidner HW (2000) Linkage of an alphavirus host range restriction to the carbohydrate-processing phenotypes of the host cell. J Gen Virol 81:161-170

2. Feng JQ, Zhang M, Mozdzanowska K, Zharikova D, Hoff H, Wunner W, Couch RB, Gerhard W (2006) Influenza A virus infection engenders a poor antibody response against the ectodomain of matrix protein 2. Virol J 3:1-13

3. Heidner HW, Johnston RE (1994) The amino-terminal residue of Sindbis virus glycoprotein E2 influences virus maturation, specific infectivity for BHK cells, and virulence in mice. J Virol 68:8064-8070

4. Heidner HW, McKnight KL, Davis NL, Johnston RE (1994) Lethality of PE2 incorporation into Sindbis virus can be suppressed by second-site mutations in E3 and E2. J Virol 68:2683-2692

5. Helenius A (1992) Unpacking the incoming influenza virus. Cell 69:577-578

6. Holsinger LJ, Lamb RA (1991) Influenza virus M2 integral membrane protein is a homotetramer stabilized by formation of disulfide bonds. Virology 183:32-43

7. Ito T, Gorman OT, Kawaoka Y, Bean WJ, Webster RG (1991) Evolutionary analysis of the influenza A virus M gene with comparisons of the M1 and M2 proteins. J Virol 65:5491-5498

8. Klimstra WB, Williams JC, Ryman KD, Heidner HW (2005) Targeting Sindbis virus-based vectors to $\mathrm{Fc}$ receptor-positive cell types. Virology 338:9-21

9. Kolpe A, Schepens B, Fiers W, Saelens X (2016) M2-based influenza vaccines: recent advances and clinical potential. Expert Rev Vaccines 16:123-136

10. Krammer F, Palese P (2015) Advances in the development of influenza virus vaccines. Nat Rev 14:167-182

11. Lamb RA, Zebedee SL, Richardson CD (1985) Influenza virus M2 protein is an integral membrane protein expressed on the infectedcell surface. Cell 40:627-633

12. London SD, Schmaljohn AL, Dalrymple JM, Rice CM (1992) Infectious enveloped RNA virus antigenic chimeras. Proc Natl Acad Sci USA 89:207-211

13. Luytjes W, Enouf V, Schipper M, Gijzen K, Liu WM, van der Lubben M, Meijer A, van der Werf S, Soethout EC (2012) HI responses induced by seasonal influenza vaccination are associated with clinical protection and with seroprotection against nonhomologous strains. Vaccine 30:5262-5269
14. Mukhopadhyay S, Zhang W, Gabler S, Chipman PR, Strauss EG, Strauss JH, Baker TS, Kuhn RJ, Rossmann MG (2006) Mapping the structure and function of the E1 and E2 glycoproteins in alphaviruses. Structure 14:63-73

15. Neirynck S, Deroo T, Saelens X, Vanlandschoot P, Jou WM, Fiers W (1999) A universal influenza A vaccine based on the extracellular domain of the M2 protein. Nat Med 5:1157-1163

16. Palese $P$ (2006) Making better influenza virus vaccines. Emerg Infect Dis 12:61-65

17. Paredes AM, Brown DT, Rothnagel R, Chiu W, Schoepp RJ, Johnston RE (1993) Three-dimensional structure of a membranecontaining virus. Proc Natl Acad Sci USA 90:9095-9099

18. Paredes AM, Heidner H, Thuman-Commike P, Prasad BVV, Johnston RE, Chiu W (1998) Structural localization of the E3 glycoprotein in attenuated Sindbis virus mutants. J Virol 72:1534-1541

19. Pinto LH, Holsinger LJ, Lamb RA (1992) Influenza virus M2 protein has ion channel activity. Cell 69:517-528

20. Reid AH, Fanning TG, Janczewski TA, McCall S, Taubenberger JK (2002) Characterization of the 1918 "Spanish" influenza virus matrix gene segment. J Virol 76:10717-10723

21. Rice CM, Strauss JH (1982) Association of Sindbis virion glycoproteins and their precursors. J Mol Biol 154:325-348

22. Ryman KD, Klimstsra WB, Nguyen KB, Biron CA, Johnston RE (2000) Alpha/beta interferon protects adult mice from fatal Sindbis virus infection and is an important determinant of cell and tissue tropism. J Virol 74:3366-3378

23. Sakaguchi T, Leser GP, Lamb RA (1996) The ion channel activity of the influenza virus M2 protein affects transport through the Golgi apparatus. J Cell Biol 133:733-747

24. Schmaljohn AL, Johnson ED, Dalrymple JM, Cole GA (1982) Non-neutralizing monoclonal antibodies can prevent lethal alphavirus encephalitis. Nature 297:70-72

25. Schnell JR, Chou JJ (2008) Structure and mechanism of the M2 proton channel of influenza A virus. Nature 451:591-595

26. Stouffer AL, Acharya R, Salom D, Levine AS, DiCostanzo L, Soto CS, Tereshko V, Nanda V, Stayrook S, DeGrado WF (2008) Structural basis for the function and inhibition of an influenza virus proton channel. Nature 451:596-599

27. Sugrue RJ, Hay AJ (1991) Structural characteristics of the M2 protein of influenza A virus: evidence that it forms a tetrameric channel. Virology 180:617-624

28. Takeuchi K, Lamb RA (1994) Influenza virus M2 protein ion channel activity stabilizes the native form of fowl plaque virus hemagglutinin during intracellular transport. J Virol 68:911-919

29. Tong S, Li Y, Rivailler P, Conrardy C, Castillo DA, Chen LM, Recuenco S, Ellison JA et al (2012) A distinct lineage of influenza A virus from bats. Proc Natl Acad Sci USA 109:4269-4274

30. Von Bonsdorff CH, Harrison SC (1975) Sindbis virus glycoproteins form a regular icosahedral lattice. J Virol 16:141-145

31. Whetter LE, MacLachlan NJ, Gebhard DH, Heidner HW, Moore PF (1989) Bluetongue virus infection of bovine monocytes. J Gen Virol 70:1663-1676

32. Zebedee SL, Lamb RA (1988) Influenza A virus M2 protein: monoclonal antibody restriction of virus growth and detection of M2 in virions. J Virol 62:2762-2772 